\title{
Clipping and STA-MCA bypass for unruptured AcomA aneurysm associated with unilateral MCA occlusion
}

Tomohiro Inoue, M.D., Hiroki Yoshida, M.D., Akira Tamura, M.D., Ph.D., and Isamu Saito, M.D., Ph.D.

Department of Neurosurgery, Fuji Brain Institute and Hospital, Shizuoka, Japan

The authors show a surgical technique of clipping in conjunction with superficial temporal artery (STA)-middle cerebral artery $(\mathrm{MCA})$ bypass to treat unruptured anterior communicating artery (AcomA) aneurysm associated with unilateral MCA occlusion. First, through MCA occlusion side, fronto-temporal craniotomy, extra-dural drilling of lesser sphenoid wing, and followed by wide exposure of Sylvian fissure, STA-MCA bypass was performed. Then, through trans-Sylvian, fronto-basal, and lateral trajectory, interhemispheric fissure was dissected from the base, which enabled good exposure and clipping of high positioned AcomA aneurysm.

The video can be found here: http://youtu.be/GWItnRSs3m4.

KEY WORDS aneurysm; anterior communicating artery; middle cerebral artery; EC-IC bypass 